\begin{tabular}{|c|l|}
\hline Title & Localization of plus one generated arrangements \\
\hline Author(s) & Palezzato, Elisa; Torielli, Michele \\
\hline Citation & $\begin{array}{l}\text { Communications in algebra, 49(1), 301-309 } \\
\text { https://doi.org/10.1080/00927872.2020.1798976 }\end{array}$ \\
\hline Issue Date & 2020-07-31 \\
\hline Doc URL & http://hdl.handle.net/2115/82340 \\
\hline Rights & $\begin{array}{l}\text { Thisis an Accepted Manuscript of an article published by Taylor \& Francis in Communications in A lgebra on 31 Jul } \\
\text { 2020, available online: http:/www.tandfonline.com/10.1080/00927872.2020.1798976. }\end{array}$ \\
\hline Type & article (author version) \\
\hline File Information & Localization of POG.pdf \\
\hline
\end{tabular}

Instructions for use 


\title{
LOCALIZATION OF PLUS-ONE GENERATED ARRANGEMENTS
}

\author{
ELISA PALEZZATO AND MICHELE TORIELLI
}

\begin{abstract}
We study the classes of free and plus-one generated hyperplane arrangements. Specifically, we describe how to compute the associated prime ideals of the Jacobian ideal of such an arrangement from its lattice of intersection. Moreover, we prove that the localization of a plus-one generated arrangement is free or plus-one generated.
\end{abstract}

Keywords: Hyperplane arrangements, Freeness, Plus-one generated arrangement, Associated prime ideal, Localization of arrangements.

MSC10: 52C35, 32S22.

\section{INTRODUCTION}

Let $V$ be a vector space of dimension $l$ over a field $\mathbb{K}$. Fix a system of coordinates $\left(x_{1}, \ldots, x_{l}\right)$ of $V^{*}$. We denote by $S=S\left(V^{*}\right)=\mathbb{K}\left[x_{1}, \ldots, x_{l}\right]$ the symmetric algebra of $V^{*}$. A hyperplane arrangement $\mathcal{A}=\left\{H_{1}, \ldots, H_{n}\right\}$ is a finite collection of hyperplanes in $V$. We refer to [6] as main reference on the theory of arrangements.

In the theory of hyperplane arrangements, the freeness is a very important algebraic property. In fact, freeness implies several interesting geometric and combinatorial properties of the arrangement itself, see [6]. By definition, an arrangement is free if and only if its module of logarithmic derivations is a free module. A lot is known about free arrangements, however there is still some mystery around the notion of freeness. For example, Terao's conjecture asserting the dependence of freeness only on the combinatorics is the longstanding open problem in this area.

In order to study this conjecture, in [1] Abe introduced the notion of plusone generated arrangement, where an arrangement is plus-one generated if and only if its module of logarithmic derivations is generated by $l+1$ elements and we can "control" their first syzygy. Moreover, Abe described how free and plus-one generated arrangements are connected. In particular, he proved that the deletion of a free arrangement is free or plus-one

Date: July 17, 2020. 
generated and vice versa, under certain additional hypothesis, if the deletion is plus-one generated then the original arrangement is free or plus-one generated.

The goal of this article is to study more in depth these two classes of arrangements. Specifically, given an arrangement $\mathcal{A}$, we describe how to compute the associated prime ideals of the module $S / J(\mathcal{A})$ from its lattice of intersection $L(\mathcal{A})$, where $J(\mathcal{A})$ is the Jacobian ideal of $\mathcal{A}$. Moreover, we prove that the localization of a plus-one generated arrangement is free or plus-one generated. Finally, we describe an example which demonstrates that the deletion of a plus-one generated arrangement is not necessarily free or plus-one generated.

All the computations in this article have been performed using the software CoCoA, see [9].

\section{PRELIMINARES ON HYPERPLANE ARRANGEMENTS}

In this section, we recall the terminology, the basic notations and some fundamental results related to hyperplane arrangements.

Let $\mathbb{K}$ be a field of characteristic zero. A finite set of affine hyperplanes $\mathcal{A}=\left\{H_{1}, \ldots, H_{n}\right\}$ in $\mathbb{K}^{l}$ is called a hyperplane arrangement. For each hyperplane $H_{i}$ we fix a defining polynomial $\alpha_{i} \in S=\mathbb{K}\left[x_{1}, \ldots, x_{l}\right]$ such that $H_{i}=\alpha_{i}^{-1}(0)$, and let $Q(\mathcal{A})=\prod_{i=1}^{n} \alpha_{i}$. An arrangement $\mathcal{A}$ is called central if each $H_{i}$ contains the origin of $\mathbb{K}^{l}$. In this case, each $\alpha_{i} \in S$ is a linear homogeneous polynomial, and hence $Q(\mathcal{A})$ is homogeneous of degree $n$. The operation of coning allows one to transform any arrangement $\mathcal{A}$ in $\mathbb{K}^{l}$ with $n$ hyperplanes into a central arrangement $c \mathcal{A}$ with $n+1$ hyperplanes in $\mathbb{K}^{l+1}$, see [6]. Unless otherwise specified, we will only consider central hyperplane arrangements. For this reason every time we will study $\mathcal{A}$ a central hyperplane arrangement, we will omit the word central.

Let $L(\mathcal{A})=\left\{\bigcap_{H \in \mathcal{B}} H \mid \mathcal{B} \subseteq \mathcal{A}\right\}$ be the lattice of intersection of $\mathcal{A}$, ordered by reverse inclusion, i.e. $X \leq Y$ if and only if $Y \subseteq X$, for $X, Y \in$ $L(\mathcal{A})$. Define a rank function on $L(\mathcal{A})$ by $\operatorname{rk}(X)=\operatorname{codim}(X) . \quad L(\mathcal{A})$ plays a fundamental role in the study of hyperplane arrangements, in fact it determines the combinatorics of the arrangement. Let $L(\mathcal{A})_{p}=\{X \in$ $L(\mathcal{A}) \mid \operatorname{rk}(X)=p\}$. We call $\mathcal{A}$ essential if $L(\mathcal{A})_{l} \neq \emptyset$.

For any flat $X \in L(\mathcal{A})$ define the localization of $\mathcal{A}$ to $X$ as the subarrangement $\mathcal{A}_{X}$ of $\mathcal{A}$ by

$$
\mathcal{A}_{X}=\{H \in \mathcal{A} \mid X \subseteq H\} .
$$

The restriction of $\mathcal{A}$ to $H \in \mathcal{A}$ is the arrangement $\mathcal{A}^{H}$ in $H \cong \mathbb{K}^{l-1}$ defined by

$$
\mathcal{A}^{H}=\left\{H \cap H^{\prime} \mid H^{\prime} \in \mathcal{A} \backslash\{H\} \text { and } H \cap H^{\prime} \neq \emptyset\right\} .
$$


We denote by $\operatorname{Der}_{\mathbb{K}^{l}}=\left\{\sum_{i=1}^{l} f_{i} \partial_{x_{i}} \mid f_{i} \in S\right\}$ the $S$-module of polynomial vector fields on $\mathbb{K}^{l}$ (or $S$-derivations). Let $\delta=\sum_{i=1}^{l} f_{i} \partial_{x_{i}} \in \operatorname{Der}_{\mathbb{K}^{l}}$. Then $\delta$ is said to be homogeneous of polynomial degree $d$ if $f_{1}, \ldots, f_{l}$ are homogeneous polynomials of degree $d$ in $S$. In this case, we write $\operatorname{pdeg}(\delta)=d$.

Definition 2.1. Let $\mathcal{A}$ be an arrangement in $\mathbb{K}^{l}$. Define the module of vector fields logarithmic tangent to $\mathcal{A}$ (or logarithmic vector fields) by

$$
D(\mathcal{A})=\left\{\delta \in \operatorname{Der}_{\mathbb{K}^{l}} \mid \delta\left(\alpha_{i}\right) \in\left\langle\alpha_{i}\right\rangle S, \forall i\right\} .
$$

The module $D(\mathcal{A})$ is obviously a graded $S$-module and we have that

$$
D(\mathcal{A})=\left\{\delta \in \operatorname{Der}_{\mathbb{K}^{l}} \mid \delta(Q(\mathcal{A})) \in\langle Q(\mathcal{A})\rangle S\right\} .
$$

In particular, since the arrangement $\mathcal{A}$ is central, then the Euler vector field $\delta_{E}=\sum_{i=1}^{l} x_{i} \partial_{x_{i}}$ belongs to $D(\mathcal{A})$, in fact $\delta_{E}(Q(\mathcal{A}))=n Q(\mathcal{A})$. In this case, we can write $D(\mathcal{A}) \cong S \cdot \delta_{E} \oplus D_{0}(\mathcal{A})$, where

$$
D_{0}(\mathcal{A})=\left\{\delta \in \operatorname{Der}_{\mathbb{K}^{l}} \mid \delta(Q(\mathcal{A}))=0\right\} .
$$

Definition 2.2. An arrangement $\mathcal{A}$ in $\mathbb{K}^{l}$ is said to be free with exponents $\left(e_{1}, \ldots, e_{l}\right)$ if and only if $D(\mathcal{A})$ is a free $S$-module and there exists a basis $\delta_{1}, \ldots, \delta_{l} \in D(\mathcal{A})$ such that $\operatorname{pdeg}\left(\delta_{i}\right)=e_{i}$, or equivalently $D(\mathcal{A}) \cong \bigoplus_{i=1}^{l} S\left(-e_{i}\right)$.

In the rest of the paper, given a tuple of integers $\left(e_{1}, \ldots, e_{l}\right)$, we will write $\left(e_{1}, \ldots, e_{l}\right)_{\leq}$, if we assume that $e_{1} \leq e_{2} \leq \cdots \leq e_{l}$.

One of the most famous characterizations of freeness is due to Saito [10] and it uses the determinant of the coefficient matrix of $\delta_{1}, \ldots, \delta_{l}$ to check if the arrangement $\mathcal{A}$ is free or not.

Theorem 2.3 (Saito's criterion). Let $\mathcal{A}$ be an arrangement in $\mathbb{K}^{l}$ and $\delta_{1}, \ldots, \delta_{l} \in$ $D(\mathcal{A})$. Then the following facts are equivalent

(1) $D(\mathcal{A})$ is free with basis $\delta_{1}, \ldots, \delta_{l}$, i. e. $D(\mathcal{A})=S \cdot \delta_{1} \oplus \cdots \oplus S \cdot \delta_{l}$.

(2) $\operatorname{det}\left(\delta_{i}\left(x_{j}\right)\right)_{i, j}=c Q(\mathcal{A})$, where $c \in \mathbb{K} \backslash\{0\}$.

(3) $\delta_{1}, \ldots, \delta_{l}$ are linearly independent over $S$ and $\sum_{i=1}^{l} \operatorname{pdeg}\left(\delta_{i}\right)=n$.

Using Saito's criterion one can prove the following result that will play an important role in Sections 4 and 5. Moreover, we will generalize it in Theorem 5.6 to the case of plus-one generated arrangements.

Theorem 2.4 ([6, Theorem 4.37]). If $\mathcal{A}$ is free, then $\mathcal{A}_{X}$ is free for any $X \in L(\mathcal{A})$.

Given an arrangement $\mathcal{A}$ in $\mathbb{K}^{l}$, the Jacobian ideal of $\mathcal{A}$ is the ideal of $S$ generated by $Q(\mathcal{A})$ and all its partial derivatives, and it is denoted by $J(\mathcal{A})$. 
$D_{0}(\mathcal{A})$ can be identified with the first syzygies of $J(\mathcal{A})$. In particular we have the following exact sequence

$$
0 \rightarrow D_{0}(\mathcal{A}) \rightarrow S(-n+1)^{l} \rightarrow J(\mathcal{A}) \rightarrow 0
$$

The Jacobian ideal has a central role in the study of free arrangements, see [6], [2] and [8] for more details. In fact, we can also characterize freeness by looking at $J(\mathcal{A})$ via the Terao's criterion. Notice that Terao described this result for characteristic 0 , but the statement holds true for any characteristic as shown in [7].

Theorem 2.5 ([11]). An arrangement $\mathcal{A}$ in $\mathbb{K}^{l}$ is free if and only if $S / J(\mathcal{A})$ is 0 or $(l-2)$-dimensional Cohen-Macaulay.

\section{Plus-one Generated ARRAngements}

In [1], the author generalized the notions of free and nearly free (see [4]) to central and essential arrangements in any dimension. However, the definition can be given also for non-essential arrangements.

Definition 3.1. Let $\mathcal{A}=\left\{H_{1}, \ldots, H_{n}\right\}$ be an arrangement in $\mathbb{K}^{l}$. We say that $\mathcal{A}$ is plus-one generated with exponents $\operatorname{PO} \exp (\mathcal{A})=\left(d_{1}, \ldots, d_{l}\right)$ and level $d$ if $D(\mathcal{A})$ has a minimal free resolution of the following form

$$
0 \longrightarrow S(-d-1) \stackrel{\left(\alpha, f_{1}, \ldots, f_{l}\right)}{\longrightarrow} S(-d) \oplus\left(\bigoplus_{i=1}^{l} S\left(-d_{i}\right)\right) \longrightarrow D(\mathcal{A}) \longrightarrow 0
$$

Remark 3.2. Let $\mathcal{A}$ be a plus-one generated arrangement in $\mathbb{K}^{l}$ with exponents $\operatorname{PO} \exp (\mathcal{A})=\left(d_{1}, \ldots, d_{l}\right)_{\leq}$and level $d$. Since $\mathcal{A}$ is central, then there exists $k \geq 2$ such that $\left(d_{1}, \ldots, d_{l}\right)_{\leq}=\left(0, \ldots, 0,1, d_{k}, \ldots, d_{l}\right)_{\leq}$. If $\mathcal{A}$ is essential, then $k=2$. If $\mathcal{A}$ is non-essential, then $k \geq 3$.

Directly from the definition, we can show the following

Lemma 3.3. Let $\mathcal{A}=\left\{H_{1}, \ldots, H_{n}\right\}$ be an arrangement in $\mathbb{K}^{l}$. $\mathcal{A}$ is plusone generated with exponents $\operatorname{PO} \exp (\mathcal{A})=\left(d_{1}, \ldots, d_{l}\right)_{\leq}$and level $d$ if and only if $S / J(\mathcal{A})$ has a minimal free resolution of the form

$0 \rightarrow S(-n-d) \rightarrow S(-n-d+1) \oplus\left(\bigoplus_{i=k}^{l} S\left(-n-d_{i}+1\right)\right) \rightarrow S(-n+1)^{l-k+2} \rightarrow S$.

Moreover, the map

$$
\partial_{3}: S(-n-d) \rightarrow S(-n-d+1) \oplus\left(\bigoplus_{i=k}^{l} S\left(-n-d_{i}+1\right)\right)
$$


is defined by a matrix of the form $\left(\alpha, f_{i_{1}}, \ldots, f_{i_{l-k+1}}\right)$, where $1 \leq i_{1}<$ $\cdots<i_{l-k+1} \leq l$. Notice that $l-k+2$ coincides with the codimension of the center of $\mathcal{A}$ or, equivalently, the rank of $\mathcal{A}$.

Proof. This equivalence follows from the fact that $D(\mathcal{A}) \cong S \cdot \delta_{E} \oplus D_{0}(\mathcal{A})$, and the fact that if $\delta_{1}, \ldots, \delta_{l} \in D_{0}(\mathcal{A})$ and there is a relation of the form $g_{0} \delta_{E}+\sum_{i=1}^{l} g_{i} \delta_{i}=0$, with $g_{i} \in S$, then $g_{0}=0$.

Example 3.4. Consider the arrangement $\mathcal{A}$ in $\mathbb{C}^{4}$ with defining polynomial $x(x-y)(x-t)(y-z)(z-t)$. It is plus-one generated with $\operatorname{PO} \exp (\mathcal{A})=$ $(1,1,2,2)$ and level 2 . In fact $D(\mathcal{A})$ has a minimal resolution of the form

$$
0 \rightarrow S(-3) \rightarrow S(-1)^{2} \oplus S(-2)^{3} \rightarrow D(\mathcal{A}) .
$$

On the other hand, the minimal resolution of $S / J(\mathcal{A})$ is

$$
0 \rightarrow S(-7) \rightarrow S(-5) \oplus S(-6)^{3} \rightarrow S(-4)^{4} \rightarrow S
$$

Example 3.5. Consider the arrangement $\mathcal{A}$ in $\mathbb{C}^{4}$ with defining polynomial $(x+y)(x-z)(x+z)(y-z)(y+z)(x-y-t)(x+y-t)(x-z-t)(x+$ $z-t)(y-z-t)(y+z-t) t$. This arrangement is plus-one generated with $\operatorname{PO} \exp (\mathcal{A})=(1,4,4,4)$ and level 5 since the minimal resolution of $S / J(\mathcal{A})$ is

$$
0 \rightarrow S(-17) \rightarrow S(-15)^{3} \oplus S(-16) \rightarrow S(-11)^{4} \rightarrow S
$$

Notice that for an arrangement $\mathcal{A}$ to be plus-one generated it is not enough that $D(\mathcal{A})$ has projective dimension 1 or equivalently that $S / J(\mathcal{A})$ has projective dimension 3 .

Example 3.6. Consider the arrangement $\mathcal{A}$ in $\mathbb{C}^{4}$ with defining polynomial $x y z t(x+y-2 z)(x-3 y+z)(-5 x+y+z)(x+y+z)$. It is not a plus-one generated arrangement since the minimal resolution of $S / J(\mathcal{A})$ is

$$
0 \rightarrow S(-13) \rightarrow S(-8) \oplus S(-11)^{3} \rightarrow S(-7)^{4} \rightarrow S
$$

In [1], Abe also described how free and plus-one generated arrangements are connected.

Theorem 3.7 ([1, Theorem 1.4]). Let $\mathcal{A}$ be an essential free hyperplane arrangement with exponents $\left(e_{1}, \ldots, e_{l}\right)$ and $H \in \mathcal{A}$. Then $\mathcal{A} \backslash\{H\}$ is free, or plus-one generated with exponents $\left(e_{1}, \ldots, e_{l}\right)$ and level $|\mathcal{A} \backslash\{H\}|-$ $\left|\mathcal{A}^{H}\right|$.

Theorem 3.8 ([1, Theorem 1.9]). Let $\mathcal{A}$ be an essential arrangement and $H \in \mathcal{A}$. Assume that $\mathcal{A} \backslash\{H\}$ is free with exponents $\left(e_{1}, \ldots, e_{l}\right)_{\leq}$. If $|\mathcal{A} \backslash\{H\}|-\left|\mathcal{A}^{H}\right| \geq e_{l-2}$, then $\mathcal{A}$ is free, or plus-one generated with $\operatorname{PO} \exp (\mathcal{A})=\left(e_{1}, \ldots, e_{l-2}, e_{l-1}+1, e_{l}+1\right)$ and level $e_{l-1}+e_{l}-|\mathcal{A}|+$ $\left|\mathcal{A}^{H}\right|+1$. 


\section{AsSOCIATED PRIME IDEALS OF $S / J(\mathcal{A})$}

Let $\mathcal{A}$ be an arrangement in $\mathbb{K}^{l}$ and $k \geq 2$. To each $X \in L(\mathcal{A})_{k}$ corresponds a prime ideal $I(X)=\left\langle\alpha_{i_{1}}, \ldots, \alpha_{i_{k}}\right\rangle$ of codimension $k$ of $S=\mathbb{K}\left[x_{1}, \ldots, x_{l}\right]$ that contains $J(\mathcal{A})$, where $X=H_{i_{1}} \cap \cdots \cap H_{i_{k}}$.

Remark 4.1. Let $\mathcal{A}$ be an arrangement in $\mathbb{K}^{l}$. There is a bijection between $L(\mathcal{A})_{2}$ and the set of associated prime ideals of $S / J(\mathcal{A})$ of codimension 2. This is because each associated prime ideal of $S / J(\mathcal{A})$ of codimension 2 corresponds to an irreducible component of the singular locus of $\mathcal{A}$. Moreover, any associated prime ideal of $S / J(\mathcal{A})$ of codimention $k$ corresponds to an element of $L(\mathcal{A})_{k}$. (For details see Section 3.8 of [5]). This gives us the following inclusions

$$
\left\{I(X) \mid X \in L(\mathcal{A})_{2}\right\} \subseteq \operatorname{Ass}_{S}(S / J(\mathcal{A})) \subseteq\left\{I(X) \mid X \in L(\mathcal{A})_{\geq 2}\right\} .
$$

Theorem 4.2. Let $\mathcal{A}$ be an arrangement in $\mathbb{K}^{l}$, and $X, Y \in L(\mathcal{A})$ such that $X \subseteq Y$.

$$
I(Y) \in \operatorname{Ass}_{S}(S / J(\mathcal{A})) \Longleftrightarrow I(Y) \in \operatorname{Ass}_{S}\left(S / J\left(\mathcal{A}_{X}\right)\right) .
$$

Proof. Consider $S_{I(X)}$ the localization of the ring $S$ by the ideal $I(X)$. We have that $J(\mathcal{A}) S_{I(X)}=J\left(\mathcal{A}_{X}\right) S_{I(X)}$. Since the ideals of $S_{I(X)}$ are in bijection with the ideal of $S$ contained in $I(X)$, by [5, Theorem 3.1], $I(Y) \in$ $\operatorname{Ass}_{S}(S / J(\mathcal{A}))$ if and only if $I(Y) S_{I(X)} \in \operatorname{Ass}_{S_{I(X)}}\left(S_{I(X)} / J(\mathcal{A}) S_{I(X)}\right)=$ $\operatorname{Ass}_{S_{I(X)}}\left(S_{I(X)} / J\left(\mathcal{A}_{X}\right) S_{I(X)}\right)$ if and only if $I(Y) \in \operatorname{Ass}_{S}\left(S / J\left(\mathcal{A}_{X}\right)\right)$.

Corollary 4.3. Let $\mathcal{A}$ be an arrangement in $\mathbb{K}^{l}$. Then

$\operatorname{Ass}_{S}(S / J(\mathcal{A})) \subseteq\left\{I(X) \mid X \in L(\mathcal{A})_{2}\right\} \cup\left\{I(X) \mid X \in L(\mathcal{A})_{\geq 3}, \mathcal{A}_{X}\right.$ non-free $\}$.

Proof. From Remark 4.1, every ideal in $\operatorname{Ass}_{S}(S / J(\mathcal{A}))$ is of the form $I(X)$ for some $X \in L(\mathcal{A})_{\geq 2}$. If $X \in L(\mathcal{A})_{\geq 3}$, then by Theorem $4.2 I(X) \in$ $\operatorname{Ass}_{S}\left(S / J\left(\mathcal{A}_{X}\right)\right)$. This implies that $S / J\left(\mathcal{A}_{X}\right)$ is not Cohen-Macaulay and hence $\mathcal{A}_{X}$ is non-free.

The previous inclusion might not be an equality in general.

Example 4.4 (c.f. [3, Example 8.5]). Consider the arrangement $\mathcal{A}$ in $\mathbb{C}^{4}$ with defining polynomial $Q(\mathcal{A})=\prod_{a=\left(a_{0}, a_{1}, a_{2}, a_{3}\right)}\left(a_{0} x_{0}+a_{1} x_{1}+a_{2} x_{2}+\right.$ $\left.a_{3} x_{3}\right)$, where $a \in\{0,1\}^{4}$ and $a \neq(0,0,0,0)$. This is plus-one generated with $\operatorname{PO} \exp (\mathcal{A})=(1,5,5,5)$ and level 5. In fact, $S / J(\mathcal{A})$ has minimal resolution

$$
0 \rightarrow S(-20) \rightarrow S(-19)^{4} \rightarrow S(-14)^{4} \rightarrow S
$$

A direct computation shows that $\operatorname{Ass}_{S}(S / J(\mathcal{A}))=\left\{I(X) \mid X \in L(\mathcal{A})_{2}\right\}$. However, if we consider $X \in L(\mathcal{A})_{4}$ the intersection of the hyperplanes $x_{1}=0, x_{2}=0, x_{3}=0, x_{4}=0$, then $\mathcal{A}_{X}=\mathcal{A}$, and hence $\mathcal{A}_{X}$ is not free. 
Since it is known by Theorem 2.4 that if $\mathcal{A}$ is a free arrangement then the localization $\mathcal{A}_{X}$ is free for any $X \in L(\mathcal{A})$, by Theorem 4.2 and Remark 4.1, we have the following.

Corollary 4.5. Let $\mathcal{A}$ be a free arrangement. Then

$$
\operatorname{Ass}_{S}(S / J(\mathcal{A}))=\left\{I(X) \mid X \in L(\mathcal{A})_{2}\right\} .
$$

Remark 4.6. By Example 4.4, the statement of Corollary 4.5 is not an equivalence.

We are now ready to state the main result of this section.

Theorem 4.7. Let $\mathcal{A}$ be an arrangement in $\mathbb{K}^{l}$ such that $S / J(\mathcal{A})$ has projective dimension 3 . Then

$\operatorname{Ass}_{S}(S / J(\mathcal{A}))=\left\{I(X) \mid X \in L(\mathcal{A})_{2}\right\} \cup\left\{I(X) \mid X \in L(\mathcal{A})_{3}, \mathcal{A}_{X}\right.$ non-free $\}$.

In particular, this holds if $\mathcal{A}$ is a plus-one generated arrangement.

Proof. By Auslander-Buchsbaum formula ([5, Theorem 19.9]), we have that $\operatorname{depth}(S / J(\mathcal{A}))=l-3$. Since the depth of a module is bounded above by the dimension of its associated prime ideals, we have that $S / J(\mathcal{A})$ cannot have associated prime ideals of codimension $k$ with $k \geq 4$. From Corollary 4.3, we have the inclusion " $\subseteq$ ". Let $X \in L(\mathcal{A})_{3}$ be such that $\mathcal{A}_{X}$ is non-free. Since $\mathcal{A}_{X}$ is an arrangement of rank $3, J\left(\mathcal{A}_{X}\right)$ coincides with its saturation with respect to the ideal $I(X)$ if and only if $\mathcal{A}_{X}$ is free, as described in the introduction of [4]. Since we assume that $\mathcal{A}_{X}$ is nonfree, this implies that $I(X)$ is an associated prime ideal of $S / J\left(\mathcal{A}_{X}\right)$. By Theorem 4.2, $I(X) \in \operatorname{Ass}_{S}(S / J(\mathcal{A}))$.

Example 4.8. Consider the hyperplane arrangement in Example 3.4. Then the associated prime ideals of $S / J(\mathcal{A})$ correspond to all rank 2 flats in $L(\mathcal{A})$ and the rank 3 flat corresponding to the intersection of the hyperplanes with equation $y=z, x=t$ and $z=t$.

Example 4.9. Consider the hyperplane arrangement in Example 3.5. Then the associated prime ideals of $S / J(\mathcal{A})$ correspond to all rank 2 flats in $L(\mathcal{A})$ and the ideals $\langle y+z, x+z, t\rangle,\langle y-z, x-z, t\rangle$, and $\langle y-t, x-t, z\rangle$.

Example 4.10. Consider the arrangement $\mathcal{A}$ in $\mathbb{C}^{4}$ with defining polynomial $t(x+y+z)(2 x+4 y+5 z)(x+4 y-5 z)(-3 x+5 y+z)(2 x+7 y+2 z)(3 x-$ $4 y+9 z)$. This is not plus-one generated because $S / J(\mathcal{A})$ has a minimal free resolution

$$
0 \rightarrow S(-11)^{3} \rightarrow S(-7) \oplus S(-10)^{5} \rightarrow S(-6)^{4} \rightarrow S .
$$

However, $S / J(\mathcal{A})$ has only $\langle x, y, z\rangle$ as embedded prime ideal. 


\section{LOCALIZATION OF PLUS-ONE GENERATED ARRANGEMENTS}

The goal of this section is to describe the localization of plus-one generated arrangements. The first step is to relate the algebraic total Betti numbers of an arrangement and the ones of its localization.

Proposition 5.1. Let $\mathcal{A}$ be an arrangement in $\mathbb{K}^{l}$ and $X \in L(\mathcal{A})$. Then for all $i \geq 0$, we have

$$
\begin{gathered}
b_{i}\left(S / J\left(\mathcal{A}_{X}\right)\right) \leq b_{i}(S / J(\mathcal{A})) \text { and } \\
b_{i}\left(D\left(\mathcal{A}_{X}\right)\right) \leq b_{i}(D(\mathcal{A})),
\end{gathered}
$$

where $b_{i}(-)$ are the algebraic total Betti numbers.

Proof. If $X \in L(\mathcal{A})$ is such that $\mathcal{A}=\mathcal{A}_{X}$, the statement is obviously true. Assume $\mathcal{A}_{X} \subsetneq \mathcal{A}$. Without loss of generalities, we can make a change of coordinates and assume that $I(X)=\left(x_{1}, \ldots, x_{s}\right)$ for some $1 \leq s \leq$ $l-1$. Consider $\mathcal{F}_{\mathcal{A}}$ a minimal free resolution of $S / J(\mathcal{A})$. As in the proof of Theorem 4.2,J(A) $S_{I(X)}=J\left(\mathcal{A}_{X}\right) S_{I(X)}$, and hence $(S / J(\mathcal{A}))_{I(X)} \cong$ $S_{I(X)} / J(\mathcal{A}) S_{I(X)} \cong S_{I(X)} / J\left(\mathcal{A}_{X}\right) S_{I(X)}$. Since the localization preserves freeness, if we localize $\mathcal{F}_{\mathcal{A}}$ at the prime ideal $I(X)$ we obtain the exact sequence $\left(\mathcal{F}_{\mathcal{A}}\right)_{I(X)}$. In general, $\left(\mathcal{F}_{\mathcal{A}}\right)_{I(X)}$ is a free resolution of $S_{I(X)} / J\left(\mathcal{A}_{X}\right) S_{I(X)}$, but it is not minimal. This implies that for all $i \geq 0$

$$
b_{i}\left(S_{I(X)} / J\left(\mathcal{A}_{X}\right) S_{I(X)}\right) \leq b_{i}(S / J(\mathcal{A})) .
$$

Let $\mathcal{F}_{\mathcal{A}_{X}}$ be a minimal free resolution of $S / J\left(\mathcal{A}_{X}\right)$. Similarly to the case of $S / J(\mathcal{A})$, we have that $\left(\mathcal{F}_{\mathcal{A}_{X}}\right)_{I(X)}$ is a free resolution of $S_{I(X)} / J\left(\mathcal{A}_{X}\right) S_{I(X)}$. Since $I(X)=\left(x_{1} \ldots, x_{s}\right)$, we have that $Q\left(\mathcal{A}_{X}\right) \in \mathbb{K}\left[x_{1} \ldots, x_{s}\right]$. This implies that all of the matrix entries of $\mathcal{F}_{\mathcal{A}_{X}}$ belong to the ideal $I(X)=$ $\left(x_{1} \ldots, x_{s}\right)$, and hence $\left(\mathcal{F}_{\mathcal{A}_{X}}\right)_{I(X)}$ is also minimal. This implies that for all $i \geq 0$

$$
b_{i}\left(S / J\left(\mathcal{A}_{X}\right)\right)=b_{i}\left(S_{I(X)} / J\left(\mathcal{A}_{X}\right) S_{I(X)}\right) \leq b_{i}(S / J(\mathcal{A})) .
$$

The second inequality follows directly from the first one and Lemma 3.3.

As a direct consequence of Proposition 5.1, we have the following result

Corollary 5.2. Let $\mathcal{A}$ be an arrangement in $\mathbb{K}^{l}$ and $X \in L(\mathcal{A})$. Then

$$
\operatorname{projdim}\left(S / J\left(\mathcal{A}_{X}\right)\right) \leq \operatorname{projdim}(S / J(\mathcal{A})) \text {. }
$$

This is equivalent to

$$
\operatorname{projdim}\left(D\left(\mathcal{A}_{X}\right)\right) \leq \operatorname{projdim}(D(\mathcal{A})) .
$$

Remark 5.3. Corollary 5.2 gives a different proof of Theorem 2.4, i.e. the fact that if $\mathcal{A}$ is free, then $\mathcal{A}_{X}$ is free for any $X \in L(\mathcal{A})$, with respect to the one that appears in [6]. 
If we also assume that $\mathcal{A}$ is a plus-one generated arrangement, Proposition 5.1 gives us the following result

Corollary 5.4. Let $\mathcal{A}$ be a plus-one generated arrangement in $\mathbb{K}^{l}$ and $X \in$ $L(\mathcal{A})$. Then $\mathcal{A}_{X}$ is free or $D\left(\mathcal{A}_{X}\right)$ is generated by $l+1$ vector fields.

Proof. If $\operatorname{rk}(X)=1$, then $\mathcal{A}_{X}=\{H\}$, for some $H \in \mathcal{A}$, and hence it is free. Assume $\operatorname{rk}(X) \geq 2$. Since $\operatorname{dim}\left(S / J\left(\mathcal{A}_{X}\right)\right)=2$, this implies that projdim $\left(S / J\left(\mathcal{A}_{X}\right)\right) \geq 2$. On the other hand, by Corollary 5.2, $\operatorname{projdim}\left(S / J\left(\mathcal{A}_{X}\right)\right) \leq \operatorname{projdim}(S / J(\mathcal{A}))=3$. This implies that, $2 \leq$ $\operatorname{projdim}\left(S / J\left(\mathcal{A}_{X}\right)\right) \leq 3$. If $\operatorname{projdim}\left(S / J\left(\mathcal{A}_{X}\right)\right)=2$, then $\mathcal{A}_{X}$ is free. Assume that $\operatorname{proj} \operatorname{dim}\left(S / J\left(\mathcal{A}_{X}\right)\right)=3$. By Proposition 5.1, $1 \leq b_{3}\left(S / J\left(\mathcal{A}_{X}\right)\right) \leq$ $b_{3}(S / J(\mathcal{A}))=1$. Moreover, by [5, Corollary 20.13], the alternating sum of the Betti numbers of $S / J\left(\mathcal{A}_{X}\right)$ is zero, and hence $S / J\left(\mathcal{A}_{X}\right)$ has a minimal free resolution of the form

$$
0 \rightarrow S \rightarrow S^{\beta} \rightarrow S^{\beta} \rightarrow S \rightarrow S / J\left(\mathcal{A}_{X}\right) \rightarrow 0,
$$

where $\beta=\operatorname{codim}(S / I(X))$. Similarly to Lemma 3.3, this implies that $D\left(\mathcal{A}_{X}\right)$ has a minimal free resolution of the form

$$
0 \rightarrow S \rightarrow S^{l+1} \rightarrow D\left(\mathcal{A}_{X}\right) \rightarrow 0
$$

and hence, $D\left(\mathcal{A}_{X}\right)$ is generated by $l+1$ vector fields.

Lemma 5.5. Let $\mathcal{A}$ be a plus-one generated arrangement in $\mathbb{K}^{l}, X \in L(\mathcal{A})$ and $\alpha \in S$ the linear form in the resolution (1). If $\alpha \notin I(X)$, then $\mathcal{A}_{X}$ is free.

Proof. Let $\mathcal{F}_{D(\mathcal{A})}$ be a minimal free resolution of $D(\mathcal{A})$ of the form (1). Since the localization preserves freeness, if we localize $\mathcal{F}_{D(\mathcal{A})}$ at the prime ideal $I(X)$ we obtain $\left(\mathcal{F}_{D(\mathcal{A})}\right)_{I(X)}$ a resolution of $D(\mathcal{A})_{I(X)}$. This implies that $b_{0}\left(D(\mathcal{A})_{I(X)}\right) \leq l+1$ and hence $D(\mathcal{A})_{I(X)}$ can be generated by $l+1$ elements. Since $\alpha \notin I(X)$, the localization of the map $\left(\alpha, f_{1}, \ldots, f_{l}\right)$ contains an invertible element (i.e. the localization of $\alpha$ ), and hence $D(\mathcal{A})_{I(X)}$ can be generated by $l$ elements. This implies that $b_{0}\left(D(\mathcal{A})_{I(X)}\right)=l$.

By the proof of Proposition 5.1, $b_{i}\left(S_{I(X)} / J(\mathcal{A}) S_{I(X)}\right)=b_{i}\left(S / J\left(\mathcal{A}_{X}\right)\right)$. This implies that $b_{i}\left(D(\mathcal{A})_{I(X)}\right)=b_{i}\left(D\left(\mathcal{A}_{X}\right)\right)$. In particular, if we consider $i=0$, we obtain that $b_{0}\left(D\left(\mathcal{A}_{X}\right)\right)=l$, and hence $\mathcal{A}_{X}$ is free.

We are now ready to prove the main result of this section.

Theorem 5.6. Let $\mathcal{A}$ be a plus-one generated arrangement in $\mathbb{K}^{l}$ and $X \in$ $L(\mathcal{A})$. Then $\mathcal{A}_{X}$ is free or plus-one generated.

Proof. Let $\mathcal{F}_{\mathcal{A}}$ be a minimal free resolution of $S / J(\mathcal{A})$ of the form (2).

If $X \in L(\mathcal{A})$ is such that $\mathcal{A}=\mathcal{A}_{X}$ or if $\mathcal{A}_{X}$ is free, the statement is obviously true. Assume $\mathcal{A}_{X} \subsetneq \mathcal{A}$ and that $\mathcal{A}_{X}$ is non-free. By Corollary 5.2, 
$\operatorname{proj\operatorname {dim}}\left(S / J\left(\mathcal{A}_{X}\right)\right)=3$. In this situation, similarly to the proof of Corollary 5.4, we have that $S / J\left(\mathcal{A}_{X}\right)$ has a minimal graded free resolution $\mathcal{F}_{\mathcal{A}_{X}}$ of the form

$0 \rightarrow S(-m-e) \rightarrow S\left(-m-d^{\prime}+1\right) \oplus\left(\bigoplus_{i=r}^{l} S\left(-m-d_{i}^{\prime}+1\right)\right) \rightarrow S(-m+1)^{l-r+2} \rightarrow S$,

where $m=\left|\mathcal{A}_{X}\right|, d^{\prime} \leq e$ and $k \leq r$. To conclude we need to show that $d^{\prime}=e$. Notice that this is equivalent to prove that the map

$$
\partial_{3}^{\prime}: S(-m-e) \rightarrow S\left(-m-d^{\prime}+1\right) \oplus\left(\bigoplus_{i=r}^{l} S\left(-m-d_{i}^{\prime}+1\right)\right)
$$

is defined by a matrix of the form $\left(\alpha^{\prime}, f_{1}^{\prime}, \ldots, f_{l-r+1}^{\prime}\right)$, where $\alpha^{\prime}, f_{i}^{\prime} \in S$ and $\alpha^{\prime}$ is zero or a homogenous polynomial of degree 1 .

By the proof of Proposition 5.1, $b_{i}\left(S_{I(X)} / J\left(\mathcal{A}_{X}\right) S_{I(X)}\right)=b_{i}\left(S / J\left(\mathcal{A}_{X}\right)\right)$. Hence,

$\operatorname{projdim}(S / J(\mathcal{A}))=\operatorname{projdim}\left(S / J\left(\mathcal{A}_{X}\right)\right)=\operatorname{projdim}\left(S_{I(X)} / J\left(\mathcal{A}_{X}\right) S_{I(X)}\right)$.

This implies that the localization of the map $\partial_{3}$ in $\mathcal{F}_{\mathcal{A}}$ is not the zero map and it is defined by the localization of the matrix $\left(\alpha, f_{i_{1}}, \ldots, f_{i_{l-k+1}}\right)$.

By Lemma 5.5, we can assume that $\alpha \in I(X)$. The localization of the map $\partial_{3}$ is defined by a matrix of the form $\left(\tilde{\alpha}, \tilde{f}_{i_{1}}, \ldots, \tilde{f}_{i_{l-k+1}}\right)$, with $\tilde{\alpha}$ not invertible. This implies that the localization of the map $\partial_{3}^{\prime}$ is represented by a matrix of the form $\left(\tilde{\alpha}, \tilde{f}_{j_{1}}, \ldots, \tilde{f}_{j_{l-r+1}}\right)$, that is a submatrix of the matrix $\left(\tilde{\alpha}, \tilde{f}_{i_{1}}, \ldots, \tilde{f}_{i_{l-k+1}}\right)$ obtained by deleting some invertible entries. As described in the proof of Proposition 5.1, we can assume that all of the matrix entries of $\mathcal{F}_{\mathcal{A}_{X}}$ belong to the ideal $I(X)$. This implies that the map $\partial_{3}^{\prime}$ is defined by a matrix of the form $\left(\alpha^{\prime}, f_{1}^{\prime}, \ldots, f_{l-r+1}^{\prime}\right)$, where $\alpha^{\prime}, f_{i}^{\prime} \in S$ and $\alpha^{\prime}$ is zero or a homogenous polynomial of degree 1 .

One of the possible next step in this study would be to understand if we can generalize Theorem 3.7 to plus-one generated arrangements. At the moment this is still an open problem. The next example shows that in general the deletion of a plus-one generated arrangement can be free, plusone generated or none of them.

Example 5.7. Consider the arrangement $\mathcal{A}$ in $\mathbb{C}^{5}$ defined by $x y z t w(x+y+$ $z)(x-w)(y+2 t)$. $\mathcal{A}$ is plus-one generated, in fact $S / J(\mathcal{A})$ has resolution

$$
0 \rightarrow S(-10) \rightarrow S(-9)^{5} \rightarrow S(-7)^{5} \rightarrow S .
$$

Consider $\mathcal{A}_{1}=\mathcal{A} \backslash\{x+y+z=0\}$. Then $\mathcal{A}_{1}$ is free, in fact $S / J\left(\mathcal{A}_{1}\right)$ has resolution

$$
0 \rightarrow S(-8)^{2} \oplus S(-7)^{2} \rightarrow S(-6)^{5} \rightarrow S
$$


Consider $\mathcal{A}_{2}=\mathcal{A} \backslash\{y+2 t=0\}$. Then $\mathcal{A}_{2}$ is plus-one generated, in fact $S / J\left(\mathcal{A}_{2}\right)$ has resolution

$$
0 \rightarrow S(-9) \rightarrow S(-8)^{4} \oplus S(-7) \rightarrow S(-6)^{5} \rightarrow S
$$

Consider $\mathcal{A}_{3}=\mathcal{A} \backslash\{x=0\}$. Then $\mathcal{A}_{3}$ is not free nor plus-one generated, in fact $S / J\left(\mathcal{A}_{3}\right)$ has resolution

$$
0 \rightarrow S(-10) \rightarrow S(-9)^{4} \rightarrow S(-8)^{7} \rightarrow S(-6)^{5} \rightarrow S
$$

Acknowledgements. The authors would like to thank M. Yoshinaga and T. N. Tran for many helpful discussions. During the preparation of this article the second author was supported by JSPS Grant-in-Aid for Early-Career Scientists (19K14493).

\section{REFERENCES}

[1] T. Abe. Plus-one generated and next to free arrangements of hyperplanes. To appear in International Mathematical Research Notices, 2019.

[2] A.M. Bigatti, E. Palezzato, and M. Torielli. New characterizations of freeness for hyperplane arrangements. Journal of Algebraic Combinatorics, 51(2):297-315, 2020.

[3] A. Dimca. Hyperplane Arrangements. Springer, 2017.

[4] A. Dimca and G. Sticlaru. Nearly free divisors and rational cuspidal curves. arXiv:1505.00666, 2015.

[5] D. Eisenbud. Commutative algebra with a view toward algebraic geometry, volume 150. Springer, 1995.

[6] P. Orlik and H. Terao. Arrangements of hyperplanes, volume 300 of Grundlehren der Mathematischen Wissenschaften [Fundamental Principles of Mathematical Sciences]. Springer-Verlag, Berlin, 1992.

[7] E. Palezzato and M. Torielli. Free hyperplane arrangements over arbitrary fields. To appear in the Journal of Algebraic Combinatorics, 2019.

[8] E. Palezzato and M. Torielli. Lefschetz properties and hyperplane arrangements. Journal of Algebra, 555:289-304, 2020.

[9] E. Palezzato and M. Torielli. Hyperplane arrangements in CoCoA. Journal of Software for Algebra and Geometry, 9(1):43-54, 2019.

[10] K. Saito. Theory of logarithmic differential forms and logarithmic vector fields. $J$. Fac. Sci. Univ. Tokyo Sect. IA Math., 27(2):265-291, 1980.

[11] H. Terao. Arrangements of hyperplanes and their freeness I. J. Fac. Sci. Univ. Tokyo Sect. IA Math., 27(2):293-312, 1980.

Elisa Palezzato, Department of Mathematics, Hokkaido University, Kita 10, Nishi 8, KitA-KU, SAPPORO 060-0810, JAPAN.

E-mail address: palezzato@math.sci.hokudai.ac.jp

Michele Torielli, Department of Mathematics, Gi-CoRE GSB, Hokkaido UNIVERSITY, KiTA 10, Nishi 8, KITA-KU, SAPPORO 060-0810, JAPAN.

E-mail address: torielli@math.sci.hokudai.ac.jp 